\title{
Influence of Microstructure on the Performance of Nitinol: A Computational Analysis
}

\author{
F.M. Weafer and M.S. Bruzzi
}

\author{
(Submitted November 1, 2013; in revised form March 11, 2014; published online April 26, 2014)
}

\begin{abstract}
One material that has found particular favor for use in biomedical endovascular stents is the near equiatomic NiTi alloy, Nitinol. One remarkable trait exhibited by this superelastic material is the improvement of its fatigue performance with increasing mean strain (Ref 1,2$)$. Clarification into this phenomenon still remains incomplete in the literature. This study proposes a microstructural explanation for this unique macroscopic behavior; it is hypothesized stress-induced martensite (SIM) will stabilize with increasing strain which, in turn, leads to the observed increase in fatigue life. Finite element analysis (FEA) is employed to investigate the behavior of a "v-strut" stent subcomponent under various strain levels. The volume fraction of SIM is analyzed to identify its potential influence on macroscopic response and, ultimately, fatigue behavior. In addition, a computational investigation is performed on the effect of crystallographic texture on macroscopic response. Granular transformational behavior is analyzed using FEA models with realistic and idealized grain structures, specifically evaluating the effect of individual grain orientations on the stress-induced martensite transformation.
\end{abstract}

Keywords fatigue, nitinol, microstructure, superelasticity

\section{Introduction}

\subsection{Medical Use of Nitinol}

Nitinol possesses a unique combination of properties which renders it suitable in a broad range of engineering applications. In particular, characteristics such as superelasticity, biocompatibility, flexibility, and compatibility with magnetic resonance imaging (MRI) procedures make it particularly appealing in the biomedical industry for use in the construction of selfexpanding endovascular stents (Ref 3). Such devices have proven effective in the treatment of atherosclerosis in a variety of vessels and arteries. However, fracture rates of up to $65.4 \%$ in such stents used in the superior femoral artery have been reported in clinical studies (Ref 4). Due to physiological movement from the cardiac systolic-diastolic cycle, in addition to the muscular movement associated with the anatomy in which they are placed, such failures can be attributed to cumulative fatigue damage. Accurate characterization of the fatigue behavior of such stents is therefore essential for their prolonged safe use in human arteries.

This article is an invited paper selected from presentations at the International Conference on Shape Memory and Superelastic Technologies 2013, held May 20-24, 2013, in Prague, Czech Republic, and has been expanded from the original presentation.

F.M. Weafer and M.S. Bruzzi, Mechanical \& Biomedical Engineering, National University of Ireland, Galway, Galway, Ireland. Contact e-mail: f.weafer1@nuigalway.ie.

\subsection{Superelasticity}

Superelasticity is the term given to the first-order phase transformation, from a parent austenite to a daughter martensite phase, which underpins Nitinol's unique performance. Nitinol's phase transformation can be induced by a change in temperature or stress; via an application of load, or upon cooling below the martensite-start temperature. Austenitic NiTi (ordered cubic B2 structure) is a hard, stiff material whereas martensitic NiTi (complex-twinned monoclinic B19' structure) is a softer, more ductile material with a lower yield stress (Ref 5). In a crystallographic context, the stress-induced martensite transformation (SIMT) occurs by rearrangement of atomic planes via Bain strain and lattice invariant shear. The twin boundaries in martensite readily shift such that the twins are predominantly oriented in one preferential direction; this process is known as de-twinning. By this microstructural process, the material can withstand approximately $6 \%$ strain without permanent deformation. This ability to accommodate such significant strains is highly desirable in stent device design for stent deliverability, durability, and conformance. Upon removal of the stress, the superelastic strain recovers at a lower stress level than at which it is induced, i.e., along a hysteresis curve (Fig. 1).

\subsection{Microstructural Texture}

As described, Nitinol derives its unique mechanical behavior from the coordinated atomic movements manifesting in a phase transformation from cubic austenite to monoclinic martensite. Therefore, any significant alignment of the atomic planes resulting from crystallographic texture can have a marked influence on mechanical response. It has been experimentally shown, crystallographic texture has a significant effect on crack trajectories in NiTi tube specimens subjected to uniaxial cyclic loading (Ref 6). In addition, significant variations in mechanical behavior have been observed between rolled and transverse directions of cold-drawn NiTi sheets 
(Ref 7); this can be attributed to the hindrance of deformed martensite structures and defects in the specimen leading to differing dominant de-twinning and reorientation modes and dislocation densities. This, in turn, may result in discrete areas of the microstructure no longer being able to transform back into austenite (stabilized SIM). Clarification into the effect of this process, with regards NiTi fatigue performance, still remains unexplored in the literature.

\subsection{Fatigue of Nitinol}

Microstructural examination of Nitinol in conjunction with in situ loading is seldom found in the literature; yet these experiments can offer a rich understanding of Nitinol's fundamental fatigue behavior. In a study by Brinson et al. (Ref 8), however, one key result is presented which offers a correlation between the micro- and macroscopic behavior of polycrystalline NiTi specimens; under low cyclic loading, localized plastic deformation is observed after as few as ten cycles (potentially being caused by the presence of stabilized SIM). The plastic deformation is observed to appear with cycling in the vicinity of the forming martensitic plates. Due to the increased localized deformation, it is observed that additional variants are formed at each cycle, providing a microscopic explanation for the macroscopically observed strain hardening. As an exciting step forward such studies begin to correlate micromechanical behavior with the overall macroscopic material response of Nitinol, however, further work in this research field is still required. Therefore, the aim of this paper was to establish a correlation between microscopic behavior, in particular the volume fraction of SIM, and the overall macroscopic material response of NiTi employing finite

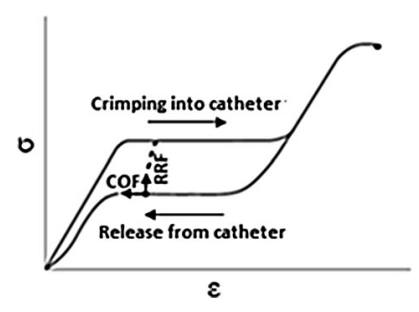

Fig. 1 Superelastic stress-strain curve portraying the stent's chronic outward force $(\mathrm{COF})$ on blood vessel in retaining it open from a stressed configuration, and radial resistive force (RRF) in resisting stent collapse. Adapted from (Ref 3) element analysis (FEA). In addition, using FEA models incorporating a granular structure, identification of possible effects of crystallographic texture on the SIMT was also investigated.

\section{Material Characterization}

\subsection{Uniaxial Material Behavior}

To investigate the effect of SIM on the fatigue behavior of a NiTi stent device, a 3D homogenous FEA model of a "v-strut" stent subcomponent was created. To accurately simulate the transformational behavior of Nitinol, an in-built material subroutine UMAT/Nitinol was employed. This material model incorporates the elastic behavior of the austentic and martensitic phases only; plasticity is neglected. To accurately characterize Nitinol's asymmetric behavior for input into the UMAT/ Nitinol, uniaxial tension-compression experimental testing was performed on linear support struts of custom manufactured specimens (Fig. 2a). The manufacturing and processing techniques used on the specimens are consistent with those performed on commercially available endovascular stents; austenite finish temperature $\left(A_{\mathrm{f}}\right)$ was found to be $11.2 \pm 2{ }^{\circ} \mathrm{C}$ using bend-free recovery method. A load-unload procedure to $6 \%$ strain is first performed prior loading to failure; this is to identify upper and lower plateau stresses (Fig. 2b). Due to the highly temperature-dependent nature of Nitinol, procedures were performed at $37^{\circ} \mathrm{C}$ using the EnduraTEC/ELF 3200 with fan-heated environmental chamber.

\subsection{Material Imaging}

To create FEA models with explicit representation of the crystallographic microstructure of polycrystalline superelastic $\mathrm{NiTi}$, microstructural geometries of suitable specimens were required. NiTi wire samples underwent a recrystallization anneal and transformational behavior was subsequently analyzed using the bend-free recovery test; $A_{\mathrm{f}}$ was found to be $1.5 \pm 0.5^{\circ} \mathrm{C}$. Specimens were subsequently manually polished and chemically etched. Finally, specimens were viewed under an optical microscope. Grain orientation distribution and average grain size were specifically identified (Fig. 3a); average grain size in the examined material was $20 \mu \mathrm{m}$. Utilizing the obtained micrographs, realistic FEA models were created which explicitly incorporated the granular microstructural
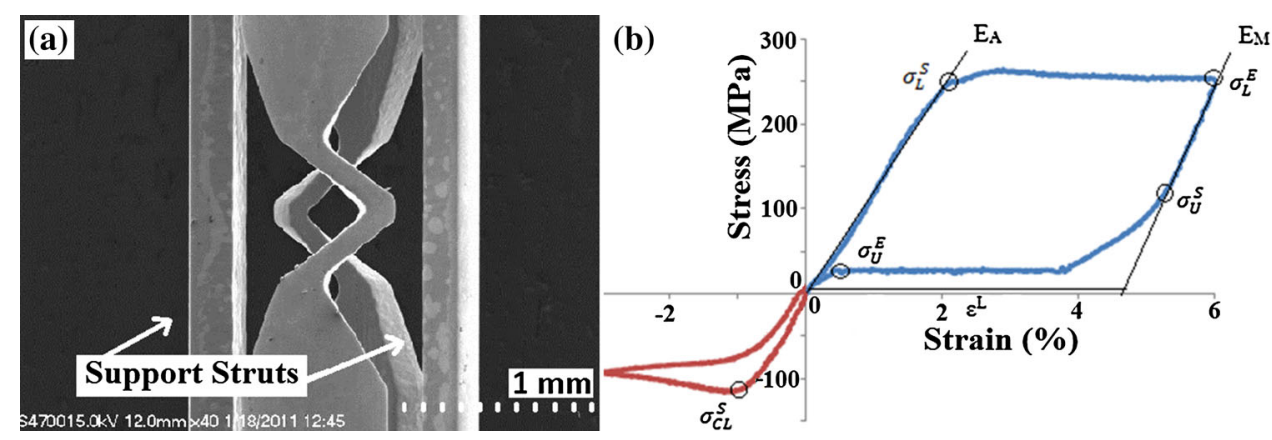

Fig. 2 (a) SEM image of "v-strut" specimen used, showing vertical support strut, (b) uniaxial stress-strain data, displaying material properties extracted for input into UMAT 

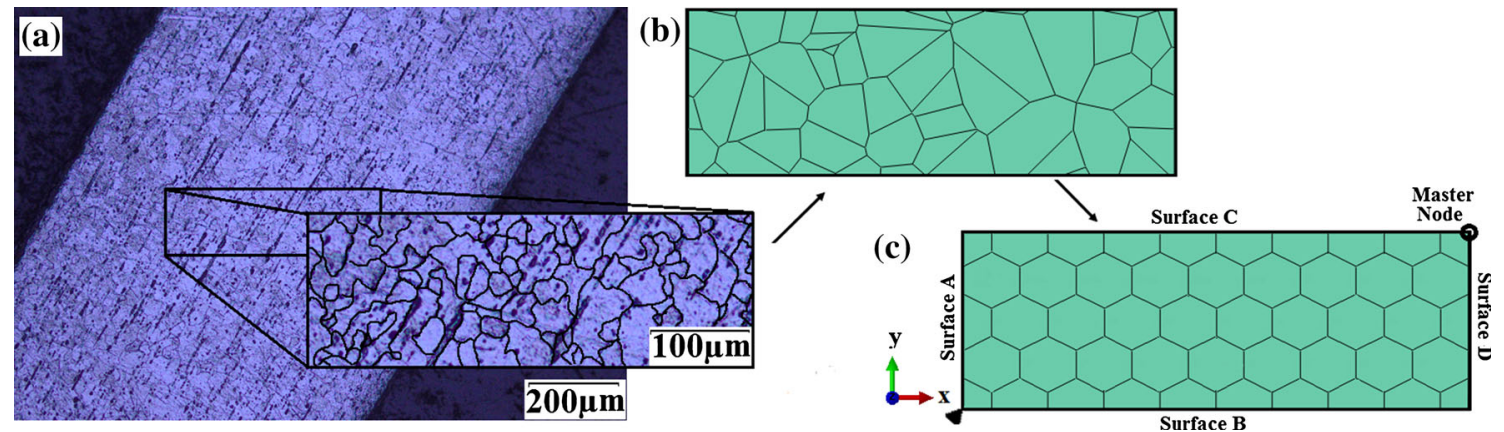

Fig. 3 (a) Experimental micrograph (b) geometric reconstruction of the microstructure, and (c) idealised geometric representation of the microstructure of superelastic austenite

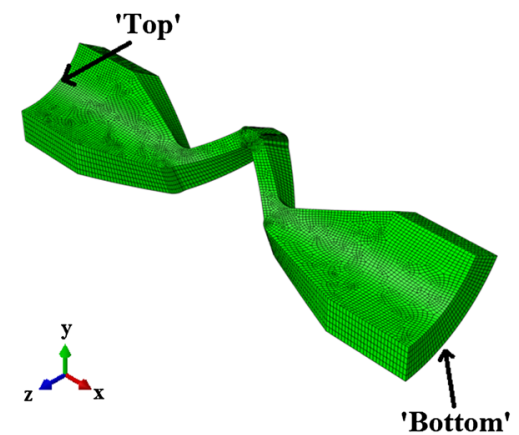

(a)

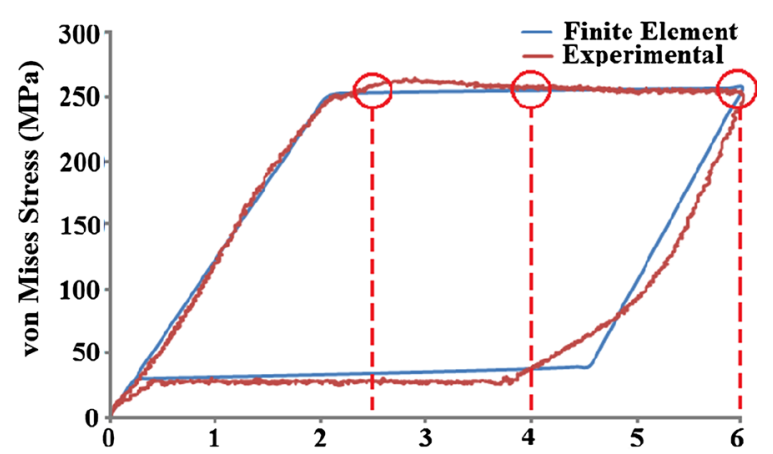

(b)

Fig. 4 (a) 3D FEA model of the "v-strut" geometry, and (b) validation of FEA model against experimental data, figure displays three strains $(2.5,4$, and $6 \%)$ targeted in this study along the superelastic plateau

nature of the superelastic austenitic material (Fig. 3b); development of these models will be discussed further in "Effect of Texture on Fatigue Behavior" section.

\section{Finite Element Model Development}

\subsection{Correlation of SIMT to Fatigue Behavior}

The potential influence of SIMT on the observed macroscopic response of Nitinol "v-strut" stent subcomponents is evaluated through identification of the volume fraction of SIM at critical locations at various strain levels in a 3D homogenous "v-strut" FEA model. The volume fraction of SIM is an explicit output, entitled "SDV21," of the UMAT/Nitinol. This value was extracted as a nodal from the node exhibiting the highest value of maximum principal tensile strain following loading to the specified strain; this node was located in the inner apex of the "v-strut" geometry. The model was constructed with 27,138 8-node hexahedral (C3D8) elements; this number of elements was deemed necessary following a convergence study. The initial boundary conditions constrained the bottom surface in all three directions $(U x=U y=U z=0)$; where $U$ is the displacement. In the first step, a boundary condition is employed to displace the "top" surface by the specified value. Simultaneously, constraining boundary conditions are placed on the nodes on the respective sides of the model to prevent out-of-plane motion $(U y=U z=0)$. Three strains spanning the experimen- tally established superelastic plateau of the specimen are targeted for analysis (namely, 2.5, 4, and 6\%) to investigate various physiologically relevant conditions experienced by a stent (Fig. 4b). It is hypothesized, at sufficiently high mean strains, SIM is present and is stabilized, and thus resulting in increased fatigue performance.

\subsection{Effect of Texture on Fatigue Behavior}

To evaluate the effect of microstructural texture on Nitinol's macroscopic response, a 2D rectangular model was created using Voronoi Tessellation based on the obtained micrographs (Fig. 3b). As the images had an aspect ratio close to 1 , it was assumed that representation of the complex realistic geometries was not necessary; idealized hexagonal units were instead used (Fig. 3c). Models were constructed using 7600 2D 8-node biquadratic plane stress quadrilateral (CPS8) elements. As symmetry was assumed, movement was restricted along surfaces $\mathrm{A}$ and $\mathrm{B}$ in the $U x$ and $U y$ directions, respectively (Fig. 3c). Surfaces C and D were constrained to replicate the $U y$ and $U x$ movement, respectively, of the master node (as shown on top right corner of model in Fig. 3c). The constitutive equations for these micro-scale models were established using single crystal data adopted from the literature (Ref 9); material properties were extracted from uniaxial tension-compression stress-strain curves for each crystallographic orientation. The grains are assumed to have homogenous behavior and the properties vary according to their crystallographic orientation to the loading direction and grain boundaries were assumed to be 


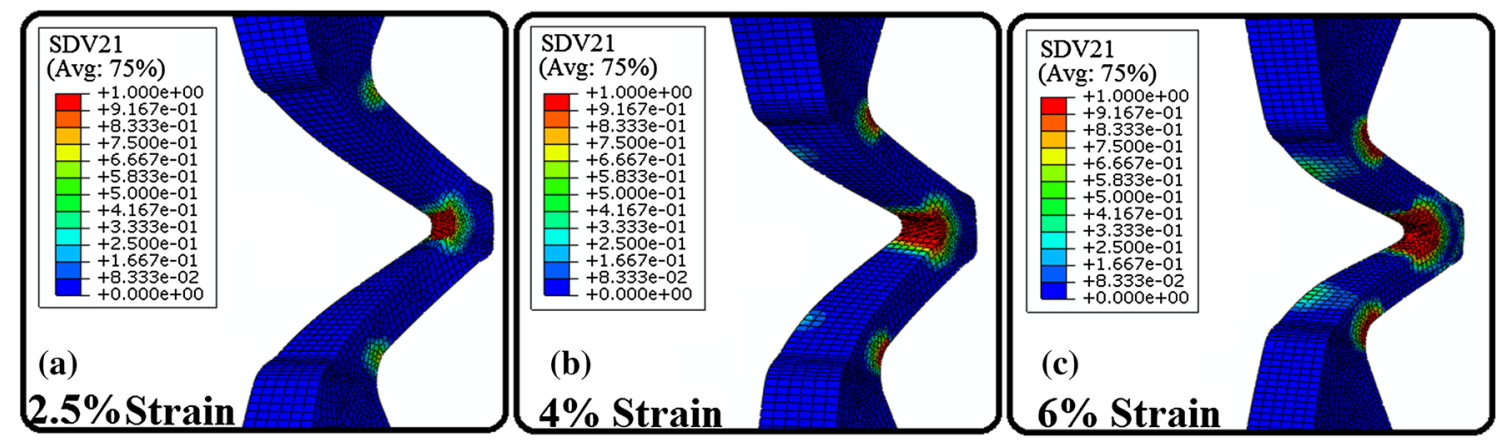

Fig. 5 Contour plots displaying the volume fraction of SIM in "v-strut" FEA model under (a) 2.5\%, (b) 4\%, and (c) 6\% compressive strain
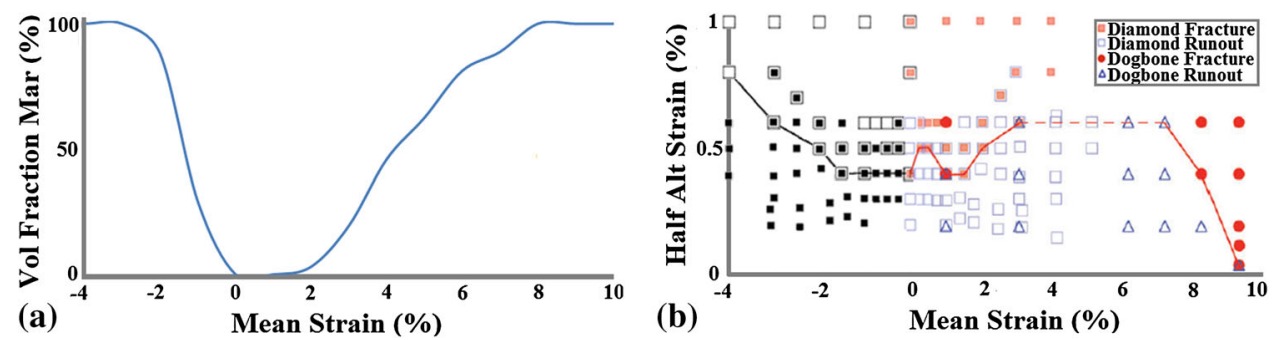

Fig. 6 Comparing (a) the volume fraction of SIM at location of max. tensile stress for various strains, and (b) the experimentally established constant life diagram for Nitinol (Ref 1,2$)$

(a)
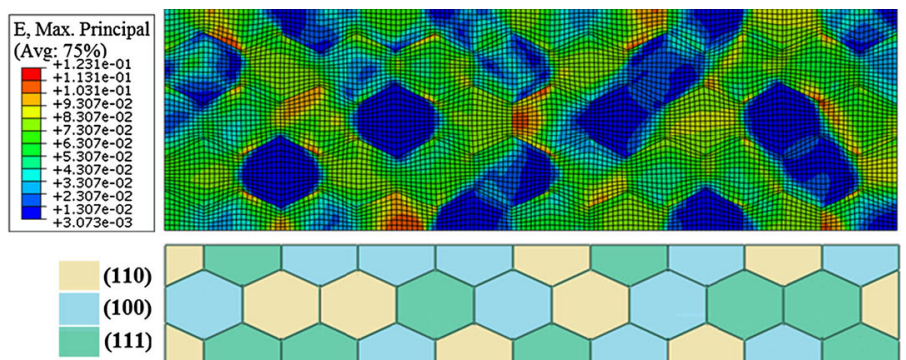

(b)
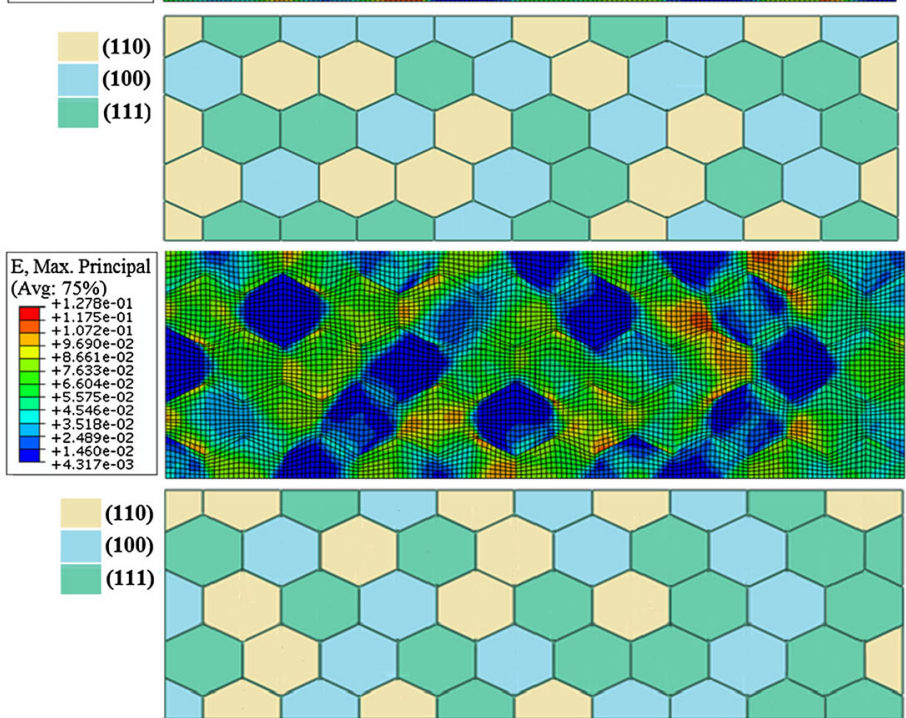
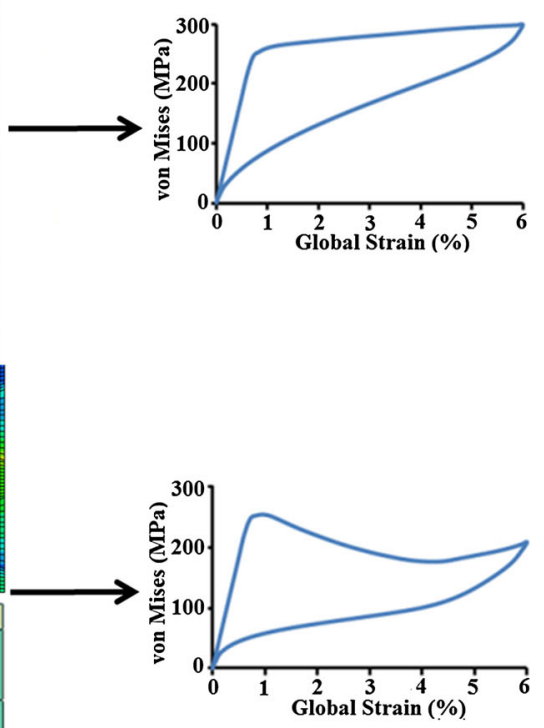

Fig. 7 Maximum principal strains and grain orientation maps of 2D idealized hexagonal granular model with two random textures, (a) and (b), deformed to $6 \%$ global strain

perfectly bonded with no inter-granular slip. Employing FEA, the effect of individual grain orientation on SIMT in randomly generated microstructural textures was also successfully inves- tigated in the complex "v-strut" geometry; this will be discussed further in "Effect of Texture on Fatigue Behavior" section of this paper. 

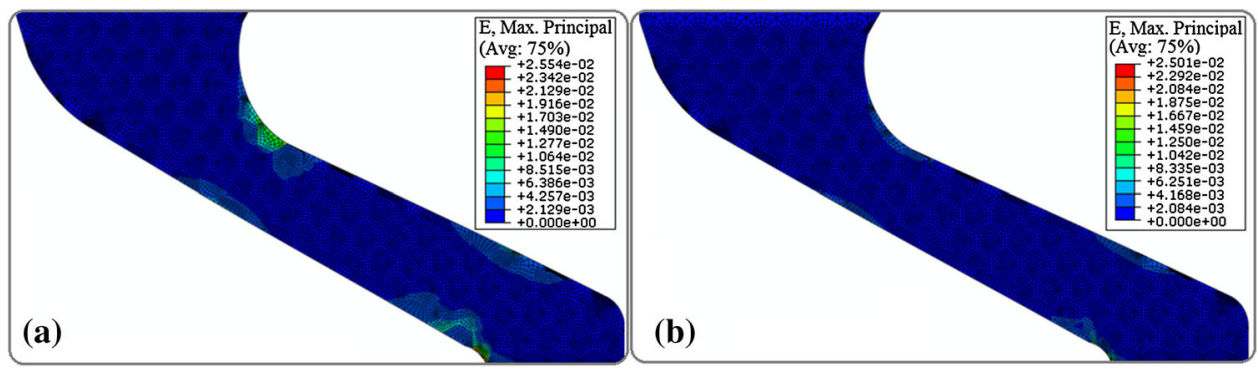

Fig. 8 Maximum principal strains in "v-strut" FEA model with two varying textures (a) and (b); peak strains in texture (a) due to the presence of (110)-orientated grains
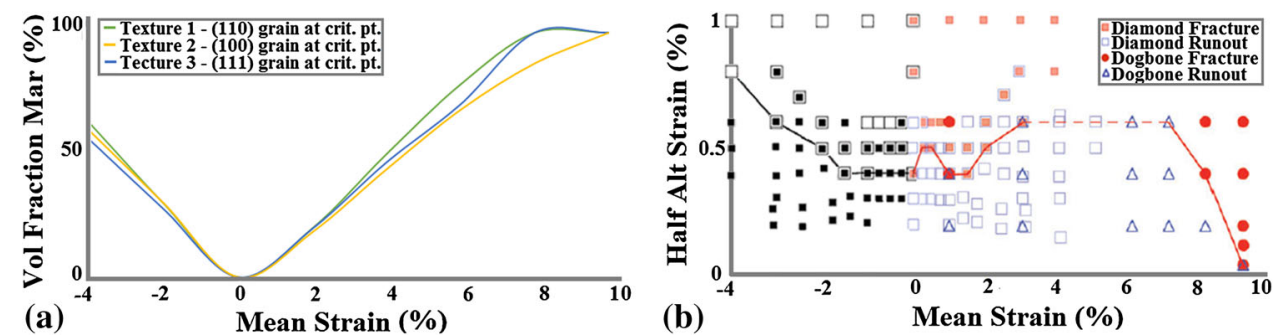

Fig. 9 Comparing (a) the volume fraction of SIM at critical location of max. tensile stress in "v-strut" model of varying microstructural crystallographic textures at various strains, and (b) the experimentally established constant life diagram for Nitinol $($ Ref 1,2$)$

\section{Results and Discussion}

\subsection{Correlation of SIMT to Fatigue Behaviour}

The highly advantageous trait exhibited by Nitinol of increased fatigue performance with increasing mean strain remains unexplained in the literature. In this paper, it is hypothesized that the increased fatigue life is attributed to the phase transformation (SIMT) experienced by the superelastic material. In this study, the volume fraction of SIM is analysed at regions of peak stress during physiologically relevant compressive loading. When assuming homogenous NiTi material properties represented within the models as a continuum, the volume fraction of SIM at region of peak stress (inner apex) is shown to increase with increasing strain (Fig. 5). However, the most significant location for analysis is that of peak tensile stress, located on the outer apex of "v-strut" geometry; as the potential site of fatigue failure initiation. The volume fraction of SIM at this critical location is found to follow a similar trend to that of the experimentally established constant life diagram for Nitinol (Ref 1, 2) (Fig. 6). Furthermore, the observed on-set of increasing fatigue performance correlates with the on-set of SIM at approximately $1.5 \%$ mean strain level which presents further evidence for the proposed hypothesis (Fig. 6).

At these critically high strain levels, deformed martensite structures and defects are hindered leading to differing dominant de-twinning, reorientation modes, and dislocation densities in discrete areas of the microstructure, resulting in the presence of stabilized SIM (Ref 7, 8). This localized strengthening of Nitinol (by means of a proposed strain hardening effect (Ref 8)), in conjunction with the self-accommodating nature of the SIM itself, may provide an explanation for the observed improved fatigue performance of Nitinol.

\subsection{Effect of Texture on Fatigue Behavior}

In applying a load-unload procedure on the idealized hexagonal rectangular models described in "Effect of Texture on Fatigue Behavior" section, it is found grains orientated in (110) crystallographic direction inhibit, while grains in (111) orientations promote the SIMT (Ref 9). This becomes evident when comparing Fig. 7(a) and 7(b); a higher volume fraction of (111)-orientated grains in model (b) results in notably lower stress values along the loading plateau due to the ease at which SIMT occurs, as compared to model (a) with more (110) grains.

This variation in transformation behavior in the individual grains results in localized stress gradients over grain boundaries; these peak stresses become particularly important when discussing fatigue behavior in the "v-strut" stent-like specimen as potential fatigue failure initiation sites. To further explore this, the influence of texture was investigated in the "v-strut" stent subcomponent; a 2D idealized hexagonal model was created for analysis incorporating the idealised hexagonal grain geometry (Fig. 8).

In analysing the volume fraction of SIM at the critical location of maximum tensile stress (located at the inner apex of "v-strut") with varying crystallographic textures, a similar trend to the experimentally established constant life diagram for Nitinol (Ref 1, 2) (Fig. 9) was observed. The three colored lines in Fig. 9a represents the stress-strain response of the "v-strut" FE model with either (100)-, (110)-, or (111)-orientated grain at the critical location (see Fig. 8). Variation in material response displayed is attributed to the different crystallographic textures generated in the "V-strut" models; in particular, varying grain orientations at critical location of maximum tensile stress impacts the macroscopic response. For example, due to the presence of (110)-orientated grains at critical location in the 
"v-strut" geometry (Fig. 8a), SIMT has been hindered and therefore localized peak strains have been induced. This may also have a role in explaining the significant scatter that is observed in experimental fatigue data.

\section{Concluding Remarks}

FEA models capable of predicting the complex constitutive behavior of Nitinol were created to determine the influence of stress-induced martensite (SIM) on the fatigue behavior of Nitinol "v-strut" stent subcomponents. As the strain levels in the specimen increase, the volume fraction of SIM correspondingly increases. The percentage of SIM at critical location of fatigue failure follows a similar trend to that of the experimentally established constant life diagram for Nitinol (Ref 1,2). Furthermore, the observed improvement of fatigue performance correlates with the on-set of SIM at approximately $1.5 \%$ mean strain. It can therefore be hypothesized, there is a strong connection relating SIM, in particular at strain levels high enough to stabilize SIM, and observed fatigue behavior.

The material model employed in this study, implemented through the in-built UMAT/Nitinol, incorporates only the elastic behavior of the austentic and martensitic phases; plasticity is neglected. This was deemed appropriate as the highest value of maximum principal strain experienced in the model following loading was $10 \%$. As the elastic limit of the material is approximately $8-9 \%$ strain, a very small percentage of local plastic deformation would be experienced by the material. The volume of material at a strain level exceeding the elastic limit of the material is very low, thus the impact on the overall strain profile of the sample will be at a minimum.

This study also successfully demonstrates the profound effect texture has on SIMT; it was demonstrated that grains orientated in (110) crystallographic direction inhibit, while grains in (111) orientations promote the SIMT progression (Ref 9). This conflict between individual granular behaviors has been shown to result in localized stress and strain gradients over grain boundaries which may previously have been neglected in simplistic homogenous FEA models. However, these induced peak tensile stresses become particularly important when discussing fatigue behavior and may present an explanation for unforeseen fatigue failures in stent devices. Therefore, in understanding the impact of local micro-structural effects on global mechanical response, it leads to a much fuller understanding of the causes of deviation of the mechanical response from predictions and unforeseen fracture in Nitinol biomedical devices.

\section{Acknowledgments}

This study was funded by Science Foundation Ireland (SFI) Research Frontiers Programme.

\section{Open Access}

This article is distributed under the terms of the Creative Commons Attribution License which permits any use, distribution, and reproduction in any medium, provided the original author(s) and the source are credited.

\section{References}

1. A.R. Pelton, V. Schroder, M.R. Mitchell, X.Y. Gong, M. Barney, and S.W. Robertson, Fatigue and Durability of Nitinol Stents, J. Mech. Behav. Biomed. Mater., 2008, 1, p 153-164

2. A.R. Pelton, X.-Y. Gong, and T. Duerig, Fatigue Testing of DiamondShaped Specimens. Proceedings of the Materials and Processes for Medical Devices Conference, 2003, p 199-204

3. B. O'Brien and M. Bruzzi, Shape Memory Alloys for Use in Medicine, Compr. Biomater., 2011, 1, p 49-72

4. D.E. Allie, C.J. Hebert, and G.M. Walker, Nitinol Stent Fractures in the SFA, Endovasc. Today, 2004, 7, p 1-8

5. Y. Liu et al., Effect of Texture Orientation on the Martensite Deformation of NiTi Shape Memory Alloy Sheet, Acta Mater, 1999, 47(2), p 645-660

6. J. Van Humbeeck and R. Stalmans, Characteristics of Shape Memory Alloys, Shape Memory Materials, K. Otsuka and C.M. Wayman, Ed., Cambridge University Press, Cambridge, 1998, p 149-183

7. S.W. Robertson, J. Stankiewicz, X.Y. Gong, and R.O. Ritchie, Cyclic Fatigue of Nitinol. Proceedings of the International Conference of Shape Memory and Superelastic Technologies, 2004, p 79-88

8. L. Brinson, Stress-Induced Transformation Behavior of a Polycrystalline NiTi Shape Memory Alloy: Micro and Macromechanical Investigations Via In Situ Optical Microscopy, J. Mech. Phys. Solids, 2004, 52(7), p 1549-1571

9. K. Gall, H. Sehitoglu, Y.I. Chumlyyakov, and I.V. Kireeva, TensionCompression Asymmetry of the Stress-Strain Response in Aged Single Crystal and Polycrystalline NiTi, Acta Metall., 1998, 47, p 1203-1217 